\title{
Design strategy and issues of the Delthaptic, a new 6-DOF parallel haptic device
}

\author{
Margot Vulliez ${ }^{\mathrm{a}}$, Said Zeghloul ${ }^{\mathrm{a}}$, Oussama Khatib ${ }^{\mathrm{b}}$ \\ ${ }^{a}$ PPRIME Institute, CNRS UPR 3346, University of Poitiers, ENSMA, 86962 Futuroscope Chasseneuil, France \\ ${ }^{b}$ Stanford Robotics Lab, A.I. Lab, Stanford University, CA 94305, USA
}

\begin{abstract}
The design of an optimal and versatile haptic device should enable human to achieve complex remote tasks in a safe and ergonomic environment. The paper presents a design strategy applied to the development of the Delthaptic, a new 6-DOF parallel haptic device. The Delthaptic is based on a coupled-parallel structure. It combines two Delta robots, linked to the two extremities of the customized ball-screw handle, to obtain its 6 degrees of freedom. The proposed haptic device provides a large and singularity-free workspace while keeping the benefits of parallel mechanisms.

The design process first consists in a phase of structural design where the mechanical architecture is defined in order to meet requirements. During a second step, the parameters of this architecture are optimized by minimizing objective functions based on desired kinematic performances. Then, a generic procedure is proposed to select the actuation with respect to dynamic capability-based criteria as well as technological aspects. Actuators and transmission systems are chosen for the Delthaptic. A prototype of the haptic device is built to experimentally assess its mechanical functioning and check the design requirements.
\end{abstract}

Keywords: Haptic device, parallel mechanism, design strategy, kinematic optimization, actuator selection.

\section{Introduction}

Collaboration between humans and robots is becoming a major research concern as human expertise is essential in manifold activity areas such as industry, medical operation, and teleoperation. In tedious or dangerous situations haptic devices allow the operator to safely achieve tasks controlling robots at distance. These systems have to be transparent and to offer a proper kinesthetic sense to their user. Therefore the design of an optimal and versatile 6-DOF haptic device constitutes a real challenge.

Several multi-purpose haptic devices are presented in the literature and some of them are commercially available. Serial devices were firstly developed with the PHANToM 6DOF of Geomagic [1], the Freedom-7 [2], the Virtuose 6D of Haption, or the Haptic Master [3]. As inertia, rigidity, and positioning accuracy represent critical matters to design transparent interfaces, fully parallel structures have been explored by some authors. Lee et al. [4] introduce a structure composed of double-chain legs. Lambert et al.[5] propose a 7-DOF parallel structure adding a grasping mobility. However these parallel devices suffer from important drawbacks: the proximity of additional singular configurations and their limited workspace. As a compromise between the specificities of parallel and serial structures, hybrid mechanisms are designed. Among them the Sigma.7 of Force Dimension, the Falcon of Novint Technologies, the compact 6-DOF device of Tsumaki et al. [6], or the Delta-R [7] combine a Delta robot [8] for translational motions and a serial wrist for rotational motions. Different new hybrid structures have been explored during the last decade, such as the 6-DOF haptic devices of Ryu et al. [9] and Lee et al. [10]. These hybrid devices provide good performances in haptic applications but have neither the rigidity and low inertia of parallel structures nor the wide workspace of serial mechanisms.

A new type of paired robots was introduced by Lallemand et al. with the 2-Delta [11]. It consists in coupling two independent mechanisms in-parallel to the end-effector to increase the mobility and the workspace of the whole robot,

Email addresses: margot.vulliez@univ-poitiers.fr (Margot Vulliez), said.zeghloul@univ-poitiers.fr (Said Zeghloul), ok@cs.stanford.edu (Oussama Khatib) 
while conserving the benefits of the basic structures. Several paired haptic devices have been developed over this past decade. Among them, the telesurgery device of Gosselin et al. [12], the Quanser $\mathrm{HD}^{2}$ [13], and the VirtuaPower [14] couple two independent serial robots, for example PHANToM devices in the $\mathrm{HD}^{2}$, to get the 5-DOF end-effector motion. The surgical haptic wand [15] is composed by two pantographs, linked in-parallel to the handle to provide five DOFs. These paired haptic devices are promising as they combine simple kinematic structures to provide a large workspace. However, besides the surgical haptic wand, the developed paired devices couple serial mechanisms, less stiff and accurate than parallel structures. None of these architectures is fully parallel with only grounded actuators. An extra active serial DOF also needs to be added to actuate the handle self-rotation. This embedded motors induce harmful mass and inertia of the device.

This paper presents the design strategy of a new 6-DOF paired parallel robot for haptic applications. This device, the Delthaptic, is composed by two Delta robots linked to the two extremities of the helical end-effector. This coupled parallel structure allows to reach a large and singularity-free workspace while keeping the benefits of parallel mechanisms such as low inertia, high dynamic performance, and high positioning accuracy.

The paper is organized as follows. The design strategy and the structure of the Delthaptic are detailed in Section 2. The geometric, kinematic, and dynamic models of the mechanism are briefly presented. Section 3 focuses on the kinematic multi-objective optimization of the geometric parameters of the structure. Then, a procedure of actuator/transmission selection is proposed in Section 4. The actuation of the Delthaptic is chosen for general purpose haptic applications. In Section 5, tests are conducted on a prototype of the Delthaptic to validate his behavior.

\section{Design strategy}

The design process of the Delthaptic aims at providing a structure suitable as versatile haptic device with 6 active DOFs. The global design strategy presented in Fig.1 is followed to develop an optimal mechanism.

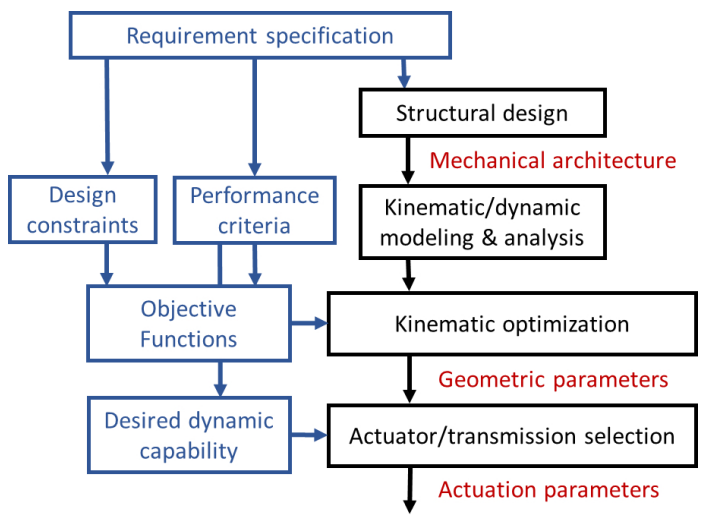

Figure 1: Design strategy scheme

The first step of the procedure consists in defining the mechanical architecture of the device with respect to the requirement specification and the literature review. The geometric parameters of the chosen structure are set through a kinematic multi-objective optimization according to desired performances and design constraints. Then, actuators and transmission systems are selected by studying the device dynamic capability along its workspace.

This Section presents the mechanical architecture of the Delthaptic and explains different technological choices made during this structural design. The kinematic and dynamic modeling of the structure is detailed.

\subsection{Structural Design}

A haptic device must meet many requirements to provide high transparency to the user. An optimal interface offers simultaneously transparency in free space, which means to enable a large and singularity-free workspace with low inertia, minimal joint clearance and friction, and transparency through contact by ensuring a large stiffness and a sufficient and adapted force feedback within a high bandwidth. These specifications are closely related to the human 
motion control capability and sense of touch. Different experiments in the literature [16, 17] have quantified human performance for the design of haptic interface. Table 1 details the desired levels of the criteria selected to design our optimal haptic device.

\begin{tabular}{ccc}
\multicolumn{3}{c}{ Table 1: Desired levels of performance } \\
Criteria & Translation & Rotation \\
\hline Workspace & $200 \times 200 \times 200 \mathrm{~mm}$ & $\pm 90 \pm 90 \pm 90^{\circ}$ \\
Position precision & $\leq 1 \mathrm{~mm}$ & $\leq 2^{\circ}$ \\
Maximum Force & $\pm 20 \mathrm{~N}$ & $\pm 0,5 \mathrm{Nm}$ \\
Force Precision & $\leq 1 \mathrm{~N}$ & $\leq 0,05 \mathrm{Nm}$ \\
\hline
\end{tabular}

To be suitable for various applications the prescribed workspace (PW) of the Delthaptic is defined according to the human hand mobilities. The amplitude of bending-extension and pronation-supination motions is around $\pm 90^{\circ}$ while the range of ulnar and radial deviations is about $\pm 45^{\circ}$. These amplitudes lead to a large and challenging prescribed rotational workspace, assimilated to a half-sphere around the wrist axis. The prescribed translational workspace is chosen with respect to the forearm range motion as a cube of $200 \times 200 \times 200 \mathrm{~mm}$.

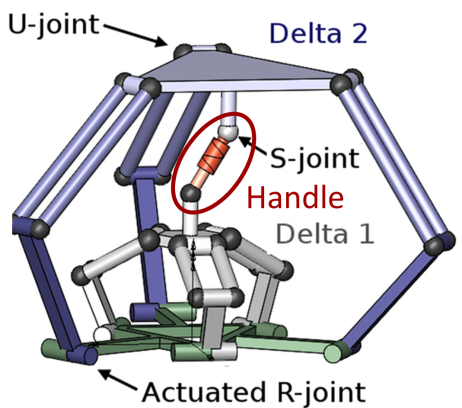

(a) Delthaptic's scheme

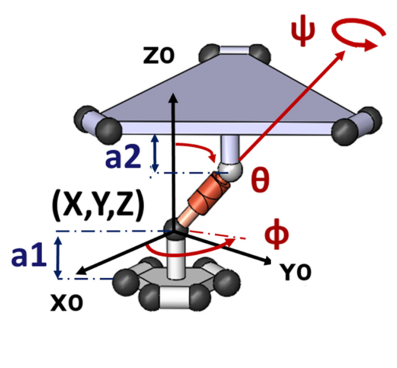

(b) Handle orientation
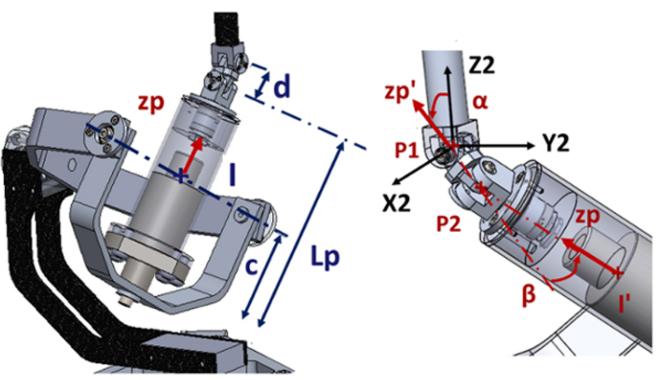

(c) Handle parameterization

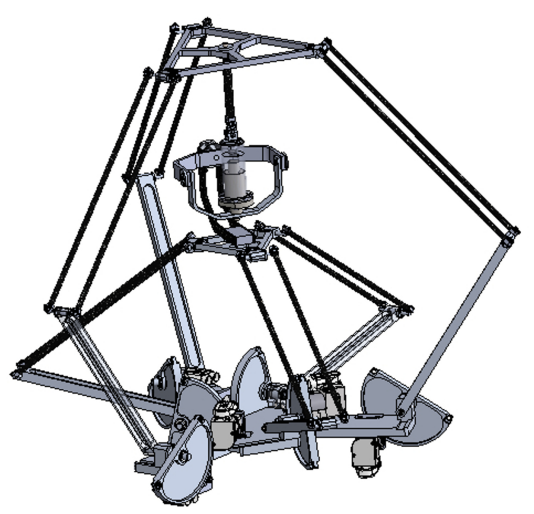

(e) Delthaptic CAD design

(d) Delta robot parameterization

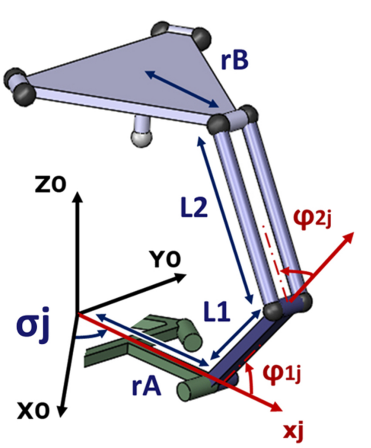

Figure 2: Structure scheme and parameterization of the Delthaptic

As high dynamics, stiffness, and accuracy are critical features of haptic device, the Delthaptic is based on a parallel structure. However traditional 6-DOF parallel manipulators have limited rotational workspace. A coupled parallel mechanism is preferred to ensure the wide prescribed workspace. The Delthaptic is composed by two Delta robots linked in-parallel to the same base platform and to the two extremities of the customized helical handle. The structure scheme of the device is presented in Fig.2. The handle upper joint with respect to the platform of the Delta 2 is a spherical joint (S-joint). A universal joint (U-joint) links the Delta 1 robot's platform to the end-effector. 
Each Delta robot $i$ is actuated by three grounded motors driving the articular coordinates $\boldsymbol{q}_{i}=\left[\varphi_{11, i} \varphi_{12, i} \varphi_{13, i}\right]^{T}$. The translational motion of the Delta 1 generates the $(X, Y, Z)$ position of the handle. The relative displacement between the two platforms results in the orientation, described by tilt-and-torsion modified Euler angles $(\phi, \theta, \psi)$. The self-rotation $\psi$ is enabled thanks to a ball screw system located inside the handle. The ball screw equation relates handle length to self-rotation: $L_{p}=L_{i n i}+\psi \times \frac{p}{2 \pi}$ with $L_{i n i}=135 \mathrm{~mm}$.

The design parameters of each Delta robot $\left(L_{1, i}, L_{2, i}, r_{i}, a_{i}\right)$ are obtained through the kinematic optimization presented in Section 3. $L_{1, i}$ and $L_{2, i}$ are the leg lengths, $r_{i}=r_{B, i}-r_{A, i}$ is the difference between the two platform radius, and $a_{i}$ is the distance between the mobile platform and the handle joint. The location angles of the Delta robots are fixed to $\sigma_{11}=60^{\circ}$ and $\sigma_{12}=0^{\circ}$ to avoid collision between the two parallel manipulators and to ensure the access to the handle.

The design of the handle is crucial to ensure the transparency of the haptic device. The user must be able to achieve 6-DOF movements without any restriction in the prescribed workspace. Moreover, these motions have to be perfectly transfer to both Delta robots through the joints between the handle and the platforms. The upper spherical joint is substituted by a 3-Rotational-joint (R-joint) serial chain to provide the large prescribed rotational motion range. The lower universal joint is replaced by a 2-DOF gimbal mechanism supporting the handle in its center of rotation, which simplifies the robot dynamics and reduces the size of the Delta 2. The CAD design of these joints appears in Fig.2 with its parameterization.

The haptic device has to transmit an accurate force feedback to the user, which is possible if the mechanism is play-free and reversible. To get a full reversibility, the thread pitch of the ball screw system needs to be large enough. It is firstly set to $p=20 \mathrm{~mm}$ for the structure dimensional synthesis but will be adjusted in Section 4 . The six degrees of freedom of the device are driven by motors equipped with absolute encoders providing a high resolution $\left(0.0014^{\circ}\right)$ compatible with the desired level of position precision. Capstan systems are designed to transmit the torque to the active joints because they are slack-free, reversible, and can possess a high reduction ratio.

The following subsections briefly present the different models of the structure. These models are valid if the geometric constraints and assumptions are respected. For instance, to ensure the expected behavior of each Delta robot, the axes of the parallelograms must be parallel, their sides of equal length, and the axes of the first and second $\mathrm{R}$-joints of each leg must be parallel. In practice, manufacturing and assembly defects could compromise these assumptions and induce infinitesimal parasitic motions of the end-effector but they are neglected in the design strategy.

\subsection{Geometric models of the Delthaptic}

The geometric models of the Delthaptic are used to determine its workspace and link the position in operational space to the articular coordinates.

The Delta robot's platform position $\boldsymbol{X}_{\boldsymbol{i}}=\left[X_{i} Y_{i} Z_{i}\right]^{T}$ is calculated from its actuated angles $\boldsymbol{q}_{\boldsymbol{i}}$ thanks to the Forward Geometric Model. Its solution $\boldsymbol{X}_{\boldsymbol{i}}$ is the intersection of three spheres (Eq. (1)) respectively accessible by each leg $j$. This intersection has two solutions, which are the upper and lower platform positions, corresponding to the two assembly modes of the Delta robot.

$$
\begin{gathered}
\left(X_{i}-x_{j, i}\right)^{2}+\left(Y_{i}-y_{j, i}\right)^{2}+\left(Z_{i}-z_{j, i}\right)^{2}=L_{2}^{2} \\
\text { with }\left\{\begin{array}{l}
x_{j, i}=\left(-r_{i}+L_{1, i} \cos \left(q_{j, i}\right)\right) \cos \left(\sigma_{j i}\right) \\
y_{j, i}=\left(-r_{i}+L_{1, i} \cos \left(q_{j, i}\right)\right) \sin \left(\sigma_{j i}\right) \\
z_{j, i}=-L_{1, i} \sin \left(q_{j, i}\right)
\end{array}\right.
\end{gathered}
$$

The Inverse Geometric Model of the Delta robot in Equation (2) evaluates the articular coordinates $\boldsymbol{q}_{i}$ needed to reach a desired Cartesian position $\boldsymbol{X}_{\boldsymbol{i}}$.

$$
\begin{gathered}
\alpha_{j, i} \cos \left(q_{j, i}\right)+\beta_{j, i} \sin \left(q_{j, i}\right)=\gamma_{j, i} \\
\left\{\begin{array}{l}
\alpha_{j, i}=2 L_{1, i}\left(-r_{i}-X_{i} \cos \left(\sigma_{j i}\right)-Y_{i} \cos \left(\sigma_{j i}\right)\right) \\
\beta_{j, i}=2 L_{1, i} Z_{i} \\
\gamma_{j, i}=2\left(-r_{i}\right)\left(X_{i} \cos \left(\sigma_{j i}\right)+Y_{i} \cos \left(\sigma_{j i}\right)\right)-r_{i}^{2}-L_{1, i}^{2}+L_{2, i}^{2}-X_{i}^{2}-Y_{i}^{2}-Z_{i}^{2}
\end{array}\right.
\end{gathered}
$$


$\boldsymbol{X}_{\boldsymbol{i}}$ is reachable if $h_{j, i}=\gamma_{j, i}^{2}-\alpha_{j, i}^{2}-\beta_{j, i}^{2} \leq 0$. The frontier equation $h_{j, i}=0$ written into the local coordinates of each leg matches the equation of a torus. The workspace of the Delta robot is given by the intersection volume of these three leg torus. $\left|h_{j, i}\right|$ represents the distance between the desired platform position $\boldsymbol{X}_{\boldsymbol{i}}$ and the workspace boundary.

Then, the geometric models of the Delthaptic device are obtained by expressing the relationships between the handle and the two Delta robots' platforms. As these relationships are the expressions of simple serial chains, the full geometric models are not developed there.

\subsection{Kinematic modeling}

The kinematic analysis of the Delthaptic is essential to ensure a proper functioning with respect to singular configurations. [18].

The kinematic model of the Delta robot is expressed in Equation (3). More details can be find on this model in

$$
\boldsymbol{A}_{\boldsymbol{i}} \dot{\boldsymbol{X}}_{\boldsymbol{i}}=\boldsymbol{B}_{i} \dot{\boldsymbol{q}}_{\boldsymbol{i}} \text { with } \boldsymbol{J}_{\boldsymbol{i}}=\boldsymbol{A}_{\boldsymbol{i}}^{-1} \boldsymbol{B}_{\boldsymbol{i}} \text { and } \boldsymbol{B}_{\boldsymbol{i}}=\operatorname{diag}\left(B_{1 i}, B_{2 i}, B_{3 i}\right)
$$

$\boldsymbol{J}_{\boldsymbol{i}}$ is the Delta $i$ Jacobian matrix, $\dot{\boldsymbol{X}}_{\boldsymbol{i}}$ the platform velocity vector and $\dot{\boldsymbol{q}}_{\boldsymbol{i}}$ the articular velocities.

The Equation (3) underlines the two assembly modes of the Delta robot, the platform upper position $\left(\operatorname{det}\left(\boldsymbol{J}_{i}\right)<0\right)$ and the lower one $\left(\operatorname{det}\left(\boldsymbol{J}_{\boldsymbol{i}}\right)>0\right)$. The Delta robot admits 8 working modes with respect to each leg posture: internal when $B_{j i}>0$ and external when $B_{j i}<0$.

The kinematic analysis of the Delthaptic is fully developed in [19]. Paired parallel structures have simple kinematic modeling by considering separately the model of each manipulator composing the device. The kinematic model of the Delthaptic, presented in Equation (4) and based on Fig. 3, can be expressed as a function of the Jacobian matrix of each Delta robot $\boldsymbol{J}_{\boldsymbol{i}}$ and a linkage matrix $\boldsymbol{J}_{\boldsymbol{c}}$. The linkage matrix relies on the kinematics of the serial chains linking the handle to both platforms.

$$
\dot{\boldsymbol{X}}=\boldsymbol{J} \dot{\boldsymbol{q}} \text { with } \boldsymbol{J}=\underbrace{\left[\begin{array}{ll}
\boldsymbol{J}_{\boldsymbol{c} 1} & \boldsymbol{J}_{\boldsymbol{c} 2}
\end{array}\right]}_{\boldsymbol{J}_{c}}\left[\begin{array}{cc}
\boldsymbol{J}_{1} & 0 \\
0 & \boldsymbol{J}_{2}
\end{array}\right]
$$

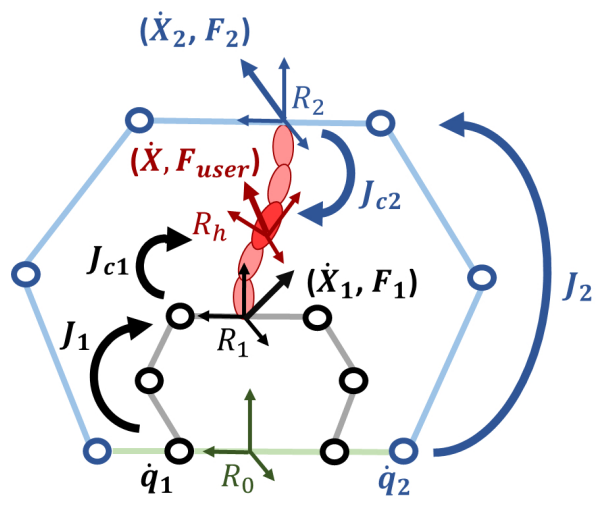

Figure 3: Delthaptic modeling scheme

The robot is into a singular configuration when the determinant of the Jacobian or the inverse Jacobian matrix vanishes. The Equation (5) demonstrates that the singularities of the haptic device are those of the two Delta robots.

$$
\operatorname{det}(\boldsymbol{J})=\operatorname{det}\left(\boldsymbol{J}_{\boldsymbol{c}}\right) \times \operatorname{det}\left(\boldsymbol{J}_{1}\right) \times \operatorname{det}\left(\boldsymbol{J}_{2}\right)
$$

The Delta robot suffers from serial singularities, when the legs are fully bended or stretched $\left(\operatorname{det}\left(\boldsymbol{B}_{i}\right)=0\right)$, and from a parallel singularity, when the three leg's parallelograms are in the plane of the platform $\left(\operatorname{det}\left(\boldsymbol{A}_{\boldsymbol{i}}\right)=0\right)$, configuration in which the platform can rotate around its axis. It is showed by writing the full kinematic Jacobian matrix of 
the Delta robot that the mechanism has three additional constraint singularities, as defined by Zlatanov [20]. These singularities occur when the geometric equation that constraints the end-effector to translational motions is no longer verified. A constraint singular configuration is reached when two of the leg's parallelograms are in the same plane $\left(\operatorname{det}\left(\boldsymbol{C}_{\boldsymbol{i}}\right)=0\right.$ with $\left.\boldsymbol{C}_{\boldsymbol{i}}=\left[\begin{array}{lll}z_{\mathbf{2 1}} & z_{\mathbf{2 2}} & z_{23}\end{array}\right]\right)$, and the platform gains a tilt mobility. There is no coupling singularity from the linkage matrix $\boldsymbol{J}_{\boldsymbol{c}}$ for this design since it is composed by simple 2-DOF gimbal and 3-R links.

\subsection{Delthaptic dynamics}

The dynamic analysis of the Delthaptic is a major design concern since a haptic device must provide high dynamics. The dynamic model is essential to choose suitable actuators and transmission systems according to desired levels of performance.

General solution for the dynamic model of parallel robots [21] has been widely studied in the literature and can easily be expressed based on the augmented object theory [22].

The handle of the Delthaptic is linked in-parallel to both Delta platforms. To provide a desired force feedback $\boldsymbol{F}_{\boldsymbol{u} \text { ser }}$ and compensate for the handle dynamics, each Delta robot has to apply the end-effector force $\boldsymbol{F}_{\boldsymbol{i}}$ expressed in Equation (6).

$$
\left[\begin{array}{l}
F_{1} \\
F_{2}
\end{array}\right]=J_{c}^{T}\left(\Lambda_{H} \ddot{X}+\mu_{H}+p_{H}+F_{u s e r}\right)
$$

In this equation $\Lambda_{H}=\Lambda_{h}+\Lambda_{u}+\Lambda_{g}$ is the kinetic energy matrix, $\boldsymbol{\mu}_{H}=\boldsymbol{\mu}_{\boldsymbol{h}}+\boldsymbol{\mu}_{\boldsymbol{u}}+\boldsymbol{\mu}_{g}$ appoints the Coriolis and Centrifugal effects, and $\boldsymbol{p}_{\boldsymbol{H}}=\boldsymbol{p}_{\boldsymbol{h}}+\boldsymbol{p}_{\boldsymbol{u}}+\boldsymbol{p}_{\boldsymbol{g}}$ represents the gravity term of the handle $\boldsymbol{h}$, the upper serial chain $\boldsymbol{u}$, and the gimbal mechanism $g$.

Then, the inverse dynamic model of the Delta robot $i$ is detailed in Equation (7) where $\boldsymbol{\Lambda}_{\boldsymbol{D}_{i}}=\sum \boldsymbol{\Lambda}_{j, i}+\boldsymbol{\Lambda}_{p_{i}}$ describes the inertia effects, $\boldsymbol{\mu}_{\boldsymbol{D}_{\boldsymbol{i}}}=\sum \boldsymbol{\mu}_{j, i}+\boldsymbol{\mu}_{\boldsymbol{p}_{i}}$ are the Coriolis and Centrifugal effects, and $\boldsymbol{p}_{\boldsymbol{D}_{\boldsymbol{i}}}=\sum \boldsymbol{p}_{j, i}+\boldsymbol{p}_{\boldsymbol{p}_{\boldsymbol{i}}}$ is the gravity term of the three legs $j, i$ and the platform $p_{i}$.

$$
\tau_{i}=J_{i}^{T}\left(\Lambda_{D_{i}} \ddot{X}_{i}+\mu_{D_{i}}+p_{D_{i}}+F_{i}\right)
$$

By combining the Equations (6) and (7) mapped into the operational space, the global inverse dynamic equation of the Delthaptic is formulated in Equation (8).

$$
\begin{gathered}
\tau=J^{T} \boldsymbol{F}_{\oplus} \\
\boldsymbol{F}_{\oplus}=\boldsymbol{\Lambda}_{\oplus} \ddot{X}+\mu_{\oplus}+p_{\oplus}+\boldsymbol{F}_{\text {user }} \\
\boldsymbol{\Lambda}_{\oplus}=\boldsymbol{\Lambda}_{H}+\underbrace{J_{c}^{-T}\left[\begin{array}{cc}
\Lambda_{D_{1}} & 0 \\
0 & \Lambda_{D_{2}}
\end{array}\right] \boldsymbol{J}_{c}^{-1}}_{\Lambda_{D}^{\oplus}} \\
\mu_{\oplus}=\mu_{H}+J_{c}^{-T}\left[\begin{array}{c}
\mu_{D_{1}} \\
\mu_{D_{2}}
\end{array}\right]-\Lambda_{D}^{\oplus} \dot{J}_{c}\left[\begin{array}{c}
\dot{X}_{1} \\
\dot{X}_{2}
\end{array}\right] \\
p_{\oplus}=p_{H}+J_{c}^{-T}\left[\begin{array}{l}
p_{D_{1}} \\
p_{D_{2}}
\end{array}\right]
\end{gathered}
$$

The dynamic modeling allows to characterize the inertial, centrifugal, Coriolis and gravity effects in the operational space of the device. The articular torques are evaluated through this equation to provide the desired force feedback to the user.

The inverse dynamic model of the Delthaptic can also be expressed into the joint space as follows in Equation (9).

$$
\begin{gathered}
\tau=\boldsymbol{A}_{\oplus} \ddot{\boldsymbol{q}}+\boldsymbol{b}_{\oplus}+\boldsymbol{G}_{\bigoplus}+\boldsymbol{J}^{T} \boldsymbol{F}_{\text {user }} \\
\text { with } \boldsymbol{A}_{\oplus}=\boldsymbol{J}^{T} \boldsymbol{\Lambda}_{\oplus} \boldsymbol{J} ; \boldsymbol{G}_{\oplus}=\boldsymbol{J}^{T} \boldsymbol{p}_{\oplus} \text { and } \boldsymbol{b}_{\oplus}=\boldsymbol{J}^{T}\left(\boldsymbol{\mu}_{\oplus}+\boldsymbol{\Lambda}_{\bigoplus} \dot{\boldsymbol{J}} \dot{\boldsymbol{q}}\right)
\end{gathered}
$$




\section{Kinematic multi-objective optimization}

The global structure of the Delthaptic has been defined in the previous section but its performances strongly depend on its dimensional synthesis. A kinematic multi-objective optimization process is implemented to determine the geometric parameters of the structure with respect to the requirement specification and the mechanism modeling.

The design vector of the Delthaptic can be defined as $\boldsymbol{I}=\left[L_{1,1}, L_{1,2}, L_{2,1}, L_{2,2}, r_{1}, r_{2}, a_{1}, a_{2}, H\right] . H$ represents the height of the center of the prescribed workspace from the base platform. The objective functions are evaluated in $n=1716$ points $P_{k}$ of the discretized prescribed workspace.

\subsection{Formulation of the problem}

Different strategies are adopted to deal with the multiple objectives during optimization process. Some authors prefer to consider successively the different objectives as single-objective optimization problems and choose arbitrarily the geometric parameters among the single-objective solutions. Others define the cost function as a weighted sum of the objectives to solve a single-objective problem. But this strategy requires a proper selection of the weight factors which needs for complex selection methods. Multi-objective optimization (MOO) process consists in finding a domain of optimal solutions, such as Pareto front or optimal parameters boxes [23]. In this paper, a MOO problem is solved using a Multi-Objective Genetic Algorithm and an optimal design is chosen on the Pareto front solution.

Many papers explore kinematic optimization and propose different criteria to fix their design vector. These kinematic performance indexes are classified in Table 2 according to their concern.

Table 2: Kinematic optimization objectives

$\begin{array}{lll}\text { Performance indexes } & \text { Objectives } & \text { Drawbacks }\end{array}$

\begin{tabular}{|c|c|c|}
\hline - Closest to the Optimal workspace [24] & Compactness & $\begin{array}{l}\text { - Depending on the distance/volume calcu- } \\
\text { lation }\end{array}$ \\
\hline - Max regular dexterous workspace [25] & Kinematic isotropy/accuracy & - Not invariant under a change of units if $\boldsymbol{J}$ \\
\hline - Max Dexterity [26] or Min $\operatorname{cond}(\boldsymbol{J})$ [27] & Distance to singularities & is not homogeneous \\
\hline - Max Global Conditioning Index [28] & Minimum error amplification & - Depending on the choice of the norm \\
\hline - Max Global kinematic Isotropy Index[29] & Static Force requirement & - No completely consistent behavior with \\
\hline - Max manipulability or Max $\operatorname{det}(\boldsymbol{J})$ & Actuator stiffness & respect to isotropy or positioning errors [30] \\
\hline $\begin{array}{l}\text { - Max volume of the stiffness hyperellipsoid } \\
\text { (SH) } \\
\text { - Min excentricity of SH } \\
\text { - Max stiffness matrix } \sigma_{\min }(\boldsymbol{K})[31]\end{array}$ & Stiffness & $\begin{array}{l}\text { - Computationally intensive } \\
\text { - Difficult stiffness modelling }\end{array}$ \\
\hline
\end{tabular}

Compulsory requirements can be introduced as constraints during the optimization procedure. In the proposed optimization process, a penalty method is adopted to transform the constrained problem into an unconstrained optimization. The design constraints that need to be verified are added as weighted penalty components $F_{C i}$ in each objective function. The penalty weight factors are chosen according to the priority order of the requirements and much more higher than the order of magnitude of the fitness component. The fitness component $F_{R i}$ of each objective function $F_{i}=F_{C}+F_{R i}$ optimizes a performance criterion. 
The five following design constraints are verified during the optimization process, for each leg $j$ of the Delta $i$, and lead to the penalty function $F_{C}$ detailed in Equation (10):

- Articular motion range: $-180^{\circ} \leq q_{j, i}\left(P_{k}\right) \leq 90^{\circ}$

- Accessibility of the PW: $h_{j, i}\left(P_{k}\right) \leq 0$

- No singularity: $\operatorname{det}\left(\boldsymbol{A}_{\boldsymbol{i}}\right) \& \operatorname{det}\left(\boldsymbol{B}_{\boldsymbol{i}}\right) \& \operatorname{det}\left(\boldsymbol{C}_{\boldsymbol{i}}\right) \neq 0 \forall\left(P_{k}\right)$

- Upper position assembly mode: $\operatorname{det}\left(\boldsymbol{J}_{\boldsymbol{i}}\left(P_{k}\right)\right)<0$

- External leg posture working mode: $B_{j i}\left(P_{k}\right)<0$

$$
\begin{aligned}
& F_{C}=F_{C 1} 10^{25}+F_{C 2} 10^{20}+F_{C 3} 10^{15}+F_{C 4} 10^{10}+F_{C 5} 10^{5} \\
& F_{C 1}=\sum_{k=1}^{n} \sum_{i=1}^{2} \sum_{j=1}^{3}\left(q_{j, i}\left(P_{k}\right)>90^{\circ} \| q_{j, i}\left(P_{k}\right)<-180^{\circ}\right) \\
& F_{C 2}=\sum_{k=1}^{n} \sum_{i=1}^{2} \sum_{j=1}^{3}\left(h_{j, i}\left(P_{k}\right)>0\right) \\
& F_{C 3}=\sum_{k=1}^{n} \sum_{i=1}^{2}\left(\operatorname{det}\left(\boldsymbol{A}_{i}\right)\left\|\operatorname{det}\left(\boldsymbol{B}_{i}\right)\right\| \operatorname{det}\left(\boldsymbol{C}_{i}\right)\left(P_{k}\right)=0\right) \\
& F_{C 4}=\sum_{k=1}^{n} \sum_{i=1}^{2}\left(\operatorname{det}\left(\boldsymbol{J}_{i}\left(P_{k}\right)\right) \geq 0\right) \\
& F_{C 5}=\sum_{k=1}^{n} \sum_{i=1}^{2} \sum_{j=1}^{3}\left(B_{j, i}\left(P_{k}\right) \geq 0\right)
\end{aligned}
$$

Two objective functions are chosen to optimize the mechanism. The first fitness function $F_{R 1}$ maximizes the distance to singular configurations. This distance can be assimilated to the dexterity of the Delta robots $\eta_{i}\left(P_{k}\right)=$ $\frac{1}{\operatorname{cond}\left(\boldsymbol{J}_{i}\left(P_{k}\right)\right)}$ and in a similar manner with respect to the constraint singularities $\kappa_{i}\left(P_{k}\right)=\frac{1}{\operatorname{cond}\left(\boldsymbol{C}_{\boldsymbol{i}}\left(P_{k}\right)\right)}$. It is worth noting that the dexterity index, based on the conditioning of the Jacobian matrix, is adapted because the studied Delta robot's Jacobian matrices are homogeneous in units. The second fitness function $F_{R 2}$ maximizes the device compactness by minimizing the distance between the workspace boundary and the prescribed workspace $\left|h_{j, i}\left(P_{k}\right)\right|$. The two objective functions are detailed in Equation (11).

$$
\begin{gathered}
F_{1, \text { SingleObj }}=F_{C}+\sum_{k=1}^{n} \sum_{i=1}^{2}\left(\operatorname{cond}\left(\boldsymbol{J}_{i}\left(P_{k}\right)\right)+\operatorname{cond}\left(\boldsymbol{C}_{\boldsymbol{i}}\left(P_{k}\right)\right)\right) \\
F_{2, \text { SingleObj }}=F_{C}+\sum_{k=1}^{n} \sum_{i=1}^{2} \sum_{j=1}^{3}\left|h_{j, i}\left(P_{k}\right)\right|
\end{gathered}
$$

For the sake of consistent evaluation of the Pareto frontier and to get comparable results, the multi-objective fitness functions are normalized according to the single-objective results as displayed in Equation (12). The single-objective optimal solution is reached when the objective function is equal to zero.

$$
F_{i, \text { Multiobj }}=F_{C}+\frac{F_{R i}-F_{i, \text { SingleObj }}^{\text {min }}}{F_{i, \text { SingleObj }}^{\text {max }}-F_{i, \text { SingleObj }}^{\text {min }}}
$$

\subsection{Results of the optimization}

The two objective functions $F_{1}$, SingleObj and $F_{2}$, SingleObj are independently optimized. The single-objective optimal solutions are considered as target values for the multi-objective process. The single-objective optimization results appear in Fig. 4. The more compact design is close to singular configurations, while the optimal solution according to dexterity results in a very large structure. 

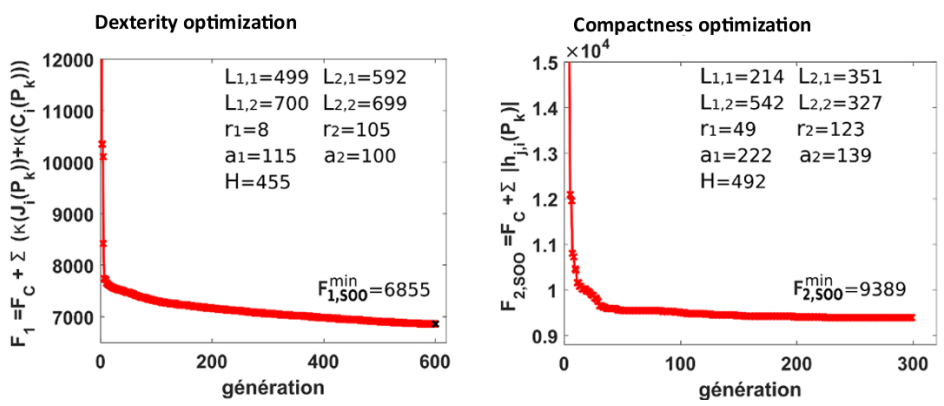

Figure 4: Single-objective optimization results

The kinematic multi-objective optimization aims at minimizing the two normalized objective functions $F_{1, M u l t i O b j}$

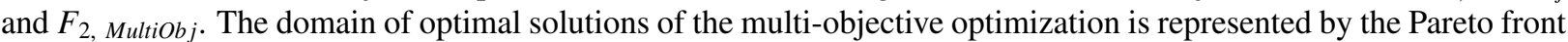
in Fig. 5. The Pareto front represents optimal compromises between the two objective functions. The single-objective optimal solutions are excluded from the multi-objective optimization results because they are unfavorable for the other objective.
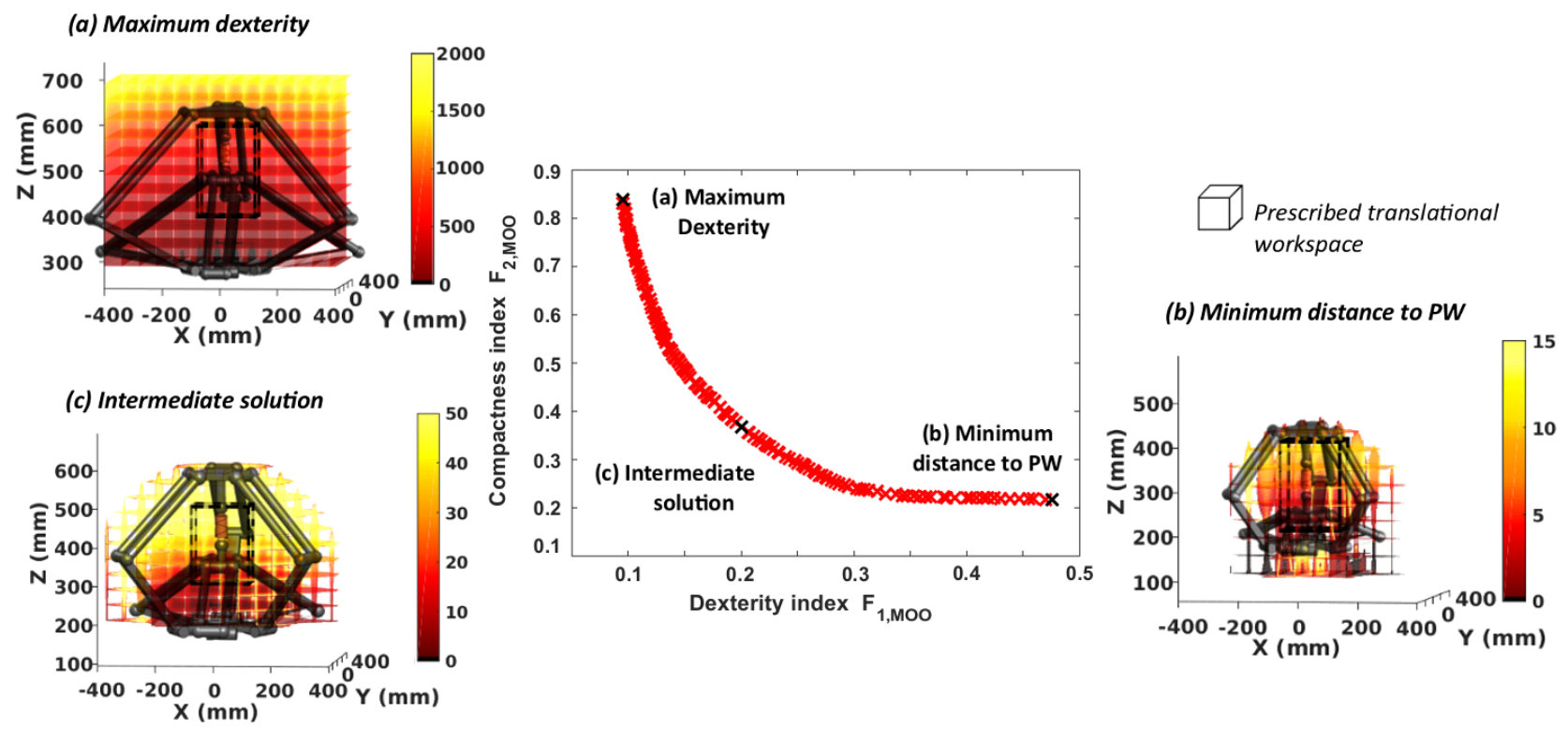

Figure 5: Pareto front of the kinematic multi-objective optimization and manipulability along the translational workspace, $|\operatorname{det}(J)|$

Different solutions can be selected by the designer from the Pareto Front: (a) has the maximum dexterity, (b) is the more compact structure respecting the prescribed workspace, and (c) is an intermediate solution. The determinant of the Jacobian matrix is represented over the constant orientation workspace at $[\phi, \theta, \psi]=[0,0,0]$ in Fig. 5. The corresponding solution is shown along with its workspace. The intermediate solution (c) is selected as optimal design since it allows to take away the singularities without significant increase of the size of the Delthaptic. This choice remains a subjective designer decision made according to his intended purpose. The vector of geometric parameters obtained thanks to the optimization process is $\boldsymbol{I}=[295,392,379,445,-23,-83,137,122,394] \mathrm{mm}$.

To validate the choice of this solution, the dexterity indexes $\eta_{i}$ and $\kappa_{i}$ are evaluated along the device's prescribed workspace for the two Delta robots. The values of these indexes are plotted in Fig. 6 over the prescribed translational workspace $([\phi, \theta, \psi]=[0,0,0])$. There is no singular configuration in the prescribed workspace boundary. The two 
Delta robots have high dexterity indexes as expected from the optimization process. The choice of an intermediate solution leads to quite homogeneous properties along the prescribed workspace.

(a) Dexterity Delta 1, $\eta_{1}$

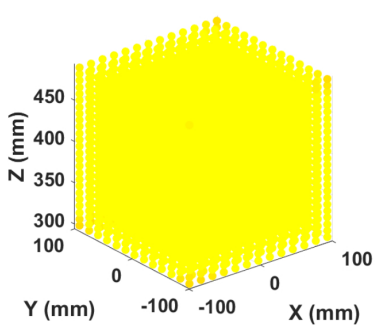

(b) Dexterity Delta $2, \eta_{2}$

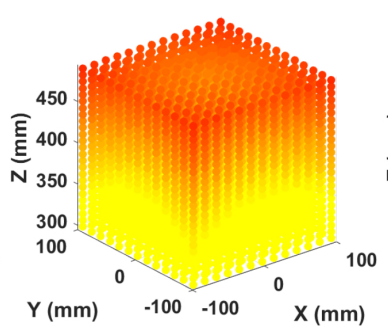

$\begin{array}{ll}\text { (c) Constraint singularity Delta } 1, \kappa_{1} & \text { (d) Constraint singularity Delta } 2, \kappa_{2}\end{array}$

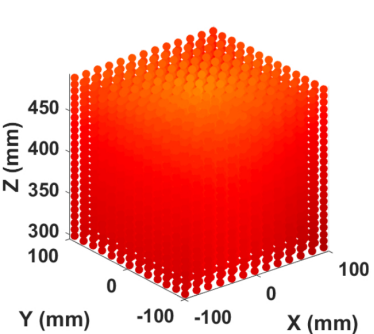

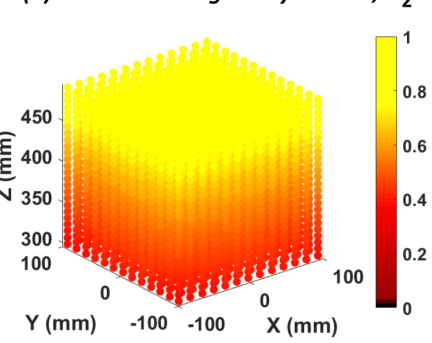

Figure 6: Dexterity analysis along the Delthaptic's prescribed translational workspace, $[\phi, \theta, \psi]=[0,0,0]$

\section{Actuator/transmission selection}

During the two first stages of the design process, the structure of the Delthaptic has been fully defined and its geometric parameters have been set according to the results of a kinematic multi-objective optimization. This strategy leads to an optimal mechanical architecture, providing high transparency and meeting the performance requirements.

This section deals with the selection of the actuators and transmission systems able to drive the mechanism and to provide the required force feedback.

\subsection{Procedure of selection}

In the literature, the actuator selection is often based on maximum joint torque requirements. In [32] the optimal choice of robot actuators aims at minimizing their mass with respect to peak torque and maximum temperature constraints on a given trajectory. Bowling and Khatib [33] introduce a method to pick the actuator out with respect to a desired level of dynamic performances, specified by the torque at performance points through the dynamic capability equation. In [34], the authors propose an optimization process to maximize the haptic device transparency by minimizing the parasitic terms in the dynamic equation. They suggest to select the motor with the smallest rotor moment of inertia which can supply the required torque along a typical path with the smallest capstan radius.

However, the only requirements of maximum torque and velocity are not sufficient to ensure a proper selection of the actuation. The effective torque along a sizing cycle has to be compared to the motor nominal torque to prevent engine overheating. In [35] and [36] optimization procedures are proposed to select drive trains by minimizing the total mass (and price) of robotic manipulators. These procedures are constrained according to motor/gearbox selection criteria recommended by the manufacturers. Criteria of nominal torque, stall torque, and maximum speed are examined to choose the motor for desired trajectories. In [35] Harmonic Drive gearboxes are selected according to their rated and maximum torques, and permissible input speed, while in [36] a constraint is set on the lifetime of the gearboxes.

It is also primordial to limit the motor/load inertia mismatch to provide a good response time and to avoid oscillations of the servo system caused by the flexibility in the coupling [37]. Fast feedback control methods [38] can provide an acceptable transient response, with low amplitude of oscillation, even in the presence of large inertia mismatch. But they involve a direct load feedback or an estimation of the load, which is complex and requires an accurate model of the system. Motor designers advice to keep the inertia ratio between the apparent inertia of the load and the rotor inertia below $20: 1$.

The following procedure is adopted to select appropriate actuators and transmission systems:

- The maximum joint torques $\tau_{\max }$ are evaluated over the prescribed workspace. For the Delthaptic, the maximum static torques are considered since the maximum force feedback is reached during stiff quasi-static contacts. The maximum velocity is not limiting for haptic device but should be evaluated for other applications. 
- The desired level of dynamic performances is defined through sets of velocity, acceleration and force in the operational space. They can be specified thanks to a typical trajectory. Highly dynamic tasks can be expected for haptic device since the human operator imposes the velocity and acceleration of the end-effector. But these dynamic motions would probably happen in free-space or without important force feedback. The required dynamic capability is strongly dependant on the application. To meet the dynamic requirements of multi-purpose haptic device, a polishing task is chosen as critical application involving both high dynamic motions and significant force feedback. The effective joint torques $\tau_{\text {eff }}$ are evaluated for this typical task in order to ensure a correct functioning of the actuation.

- A couple of actuator and transmission system is chosen for each active joint by checking three selection criteria: The speed-torque characteristic of the motor covers the required maximum torque $\tau_{\max }$ and velocity. The motor nominal torque $C_{m o t}^{\text {nom }}$ is superior to the effective torque $\tau_{\text {eff }}$ along the dynamic sizing trajectory. The rotor inertia $J_{m o t}$ is superior or equal to the apparent inertia of the load $J_{e q}$ over 20.

\subsection{Static force capability}

The static force capability of the haptic device is evaluated for six different values of the thread pitch $p=$ $[40,60,80,100,120,140] \mathrm{mm}$.

The static Equation (13) of the Delthaptic is bounded with respect to maximum joint torques fixed to $\tau_{\max }=10 \mathrm{Nm}$. The maximum force and torque which can be rendered to the user are evaluated thanks to the inequality (Eq. (14)), where translational and rotational feedback are considered separately.

$$
\begin{aligned}
& \boldsymbol{\tau}=\boldsymbol{J}^{T}\left(\boldsymbol{p}_{\oplus}+\boldsymbol{F}_{\text {user }}\right) \\
& \underline{\boldsymbol{\Gamma}} \leq \boldsymbol{J}_{\boldsymbol{f} \boldsymbol{T}}\left[\begin{array}{c}
\boldsymbol{F}_{\boldsymbol{x}} \\
\boldsymbol{F}_{\boldsymbol{y}} \\
\boldsymbol{F}_{\boldsymbol{z}}
\end{array}\right]+\boldsymbol{J}_{\boldsymbol{c} \boldsymbol{T}}\left[\begin{array}{c}
\boldsymbol{C}_{\boldsymbol{x}} \\
\boldsymbol{C}_{\boldsymbol{y}} \\
\boldsymbol{C}_{z}
\end{array}\right] \leq \overline{\boldsymbol{\Gamma}} \text { with } \begin{array}{c}
\overline{\boldsymbol{\Gamma}}=-\left[\boldsymbol{\tau}_{\max }\right]-\boldsymbol{J}^{T} \boldsymbol{p}_{\oplus} \\
\overline{\boldsymbol{\Gamma}}=\left[\boldsymbol{\tau}_{\max }\right]-\boldsymbol{J}^{T} \boldsymbol{p}_{\oplus} \\
\boldsymbol{J}^{T}=\left[\begin{array}{ll}
\boldsymbol{J}_{\boldsymbol{f}} \boldsymbol{J} & \boldsymbol{J}_{\boldsymbol{c} \boldsymbol{T}}
\end{array}\right]
\end{array}
\end{aligned}
$$

The static force and torque ellipsoids at the center of the prescribed workspace $[X, Y, Z, \phi, \theta, \psi]=[0,0, H, 0,0,0]$ are evaluated. Since the thread pitch has a very small influence on the static force capability, the force ellipsoid is only displayed for $p=120 \mathrm{~mm}$ in Fig. 7 .

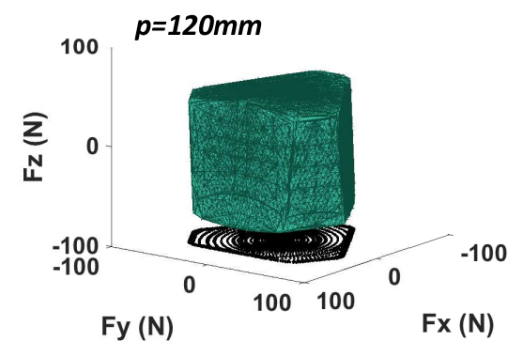

Figure 7: Static force ellipsoid at $[X, Y, Z, \phi, \theta, \psi]=[0,0, H, 0,0,0]$

The static torque capability in Fig. 8 shows that a minimum thread pitch $p=100 \mathrm{~mm}$ is necessary for the ballscrew system to get quite isotropic torque feedback. A smaller thread pitch would lead to the need for important motor torques to provide the torque feedback about the handle axis. An optimal thread pitch $p=120 \mathrm{~mm}$ is chosen for the design to reduce the maximum joint torques.

The static torques which render the maximum required force feedback are evaluated over the prescribed workspace. The desired maximum force and torque in operational space are $20 \mathrm{~N}$ and $0.5 \mathrm{Nm}$ (Table 1). The maximum torques are calculated thanks to the Equation (13). Due to the symmetric structure of the Delta robot, it has been chosen to select 

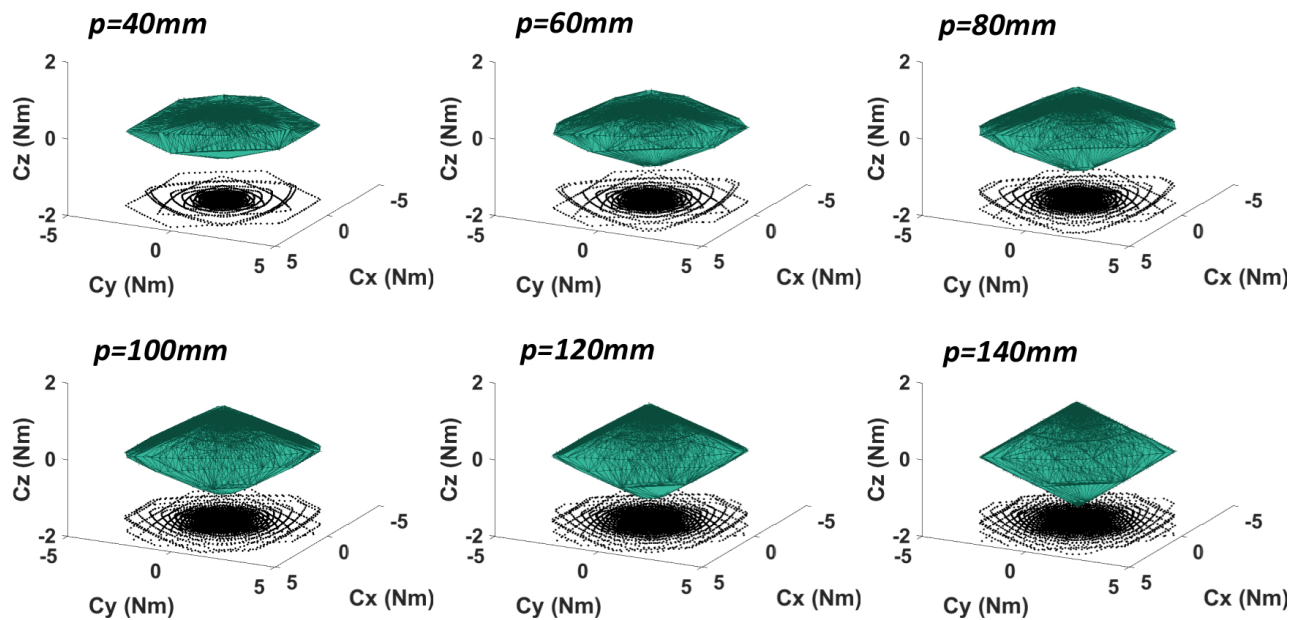

Figure 8: Static torque ellipsoids at $[X, Y, Z, \phi, \theta, \psi]=[0,0, H, 0,0,0]$

Table 3: Maximum static torque for the required force feedback

\begin{tabular}{ccccccc} 
Force & $F_{x}$ & $F_{y}$ & $F_{z}$ & $C_{x}$ & $C_{y}$ & $C_{z}$ \\
feedback & $20 N$ & $20 N$ & $20 N$ & $0.5 N m$ & $0.5 N m$ & $0.5 N m$ \\
\hline$\tau_{\max , \text { Delta } 1}[\mathrm{Nm}]$ & 7.94 & 8.56 & 8.08 & 8.08 & 7.54 & 6.57 \\
$\tau_{\max , \text { Delta } 2}[\mathrm{Nm}]$ & 9.94 & 9.76 & 11.01 & 12.91 & 12.59 & 9.28 \\
\hline
\end{tabular}

the same motor for the three axes. The maximum static torque for each Delta robot and each required force feedback are evaluated in Table 3 over the prescribed workspace.

The maximum required torque over the whole prescribed workspace is $\tau_{\max , \text { Delta1 }}=9 \mathrm{Nm}$ for the Delta 1 and $\tau_{\max , \text { Delta } 2}=13 \mathrm{Nm}$ for the Delta 2.

\subsection{Desired dynamic performance}

A typical sizing trajectory of polishing, combining high dynamic motions and force feedback, is defined to characterize the desired level of dynamic performance of the haptic device.
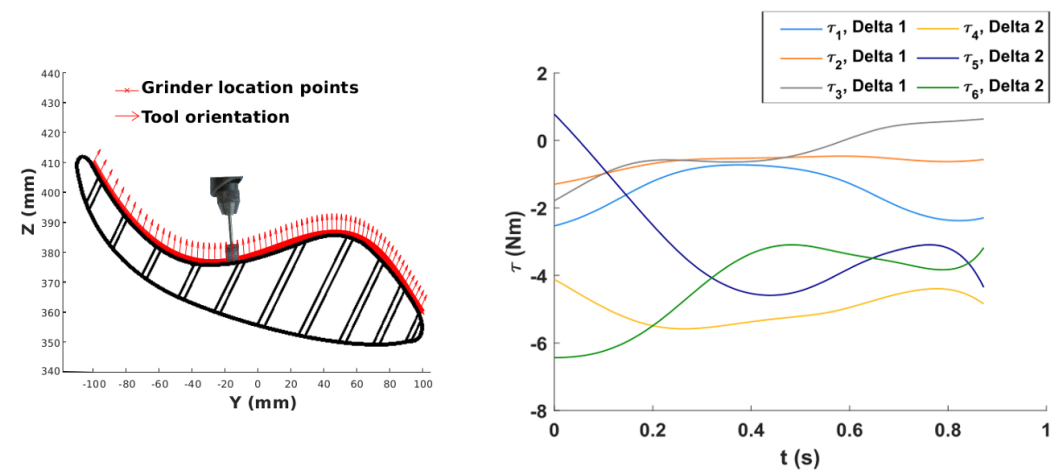

Figure 9: Joint torques along a polishing trajectory

It is assumed that the operator follows a polishing path on a turbine blade in the plane $(Y Z)$, represented on the Fig. 9 by the grinder location and orientation. The tool stays normal to the surface along the path. The trajectory is interpolated from the path at a feedrate $f=0.25 \mathrm{~m} / \mathrm{s}$ without considering acceleration or jerk limits as the human 
kinematic capabilities are very high. The resulting articular trajectories are computed through the Inverse Kinematic Model of the Delthaptic. A normal force feedback $F_{n}=5 \mathrm{~N}$, a tangential force feedback $F_{t}=2 N$, and a self-rotational torque feedback $C_{n}=0.1 \mathrm{Nm}$ are considered for this application.

The joint torques along this sizing trajectory are evaluated according to the dynamic equation (Eq. (8)) and are displayed in Fig. 9. The evolution of these dynamic torques is conform to the maximum required torques defined in the previous subsection.

The effective joint torques are evaluated thanks to the Equation (15) for this typical trajectory.

$$
\tau_{e f f}=\sqrt{\frac{\sum\left(\tau_{i}^{2} \times d t_{i}\right)}{t_{t o t}}}
$$

$\tau_{e f f, 1}=1.61 \mathrm{Nm}, \tau_{e f f, 2}=0.68 \mathrm{Nm}, \tau_{e f f, 3}=0.7 \mathrm{Nm}, \tau_{e f f, 4}=5.03 \mathrm{Nm}, \tau_{e f f, 5}=3.42 \mathrm{Nm}$, and $\tau_{e f f, 6}=4.42 \mathrm{Nm}$.

It has been chosen to select the three same couples of actuators and transmission systems for each Delta robot. The maximum effective torques are therefore retained for the two Delta: $\tau_{\text {eff,Delta } 1}=1.61 \mathrm{Nm}$ and $\tau_{\text {eff,Delta2 }}=5.03 \mathrm{Nm}$.

\subsection{Choice of the actuation}

Poole et al. [39] present a methodology to select the best actuator technology and configuration using three main figures of merit on performance (force, stroke and frequency) together with the analysis of the user requirements. Haptic applications request accurate joint torques in a large bandwidth, even during very slow motions. Electric engines are chosen to actuate the Delthaptic. In direct torque control, brushless synchronous motors suffer from undesired torque ripple coming from discontinuities in their electromagnetic flux. Different methods to reduce this phenomenon are proposed in the literature [40,41]. But they are complex control strategies and would affect the performances of the force feedback. Other motor technologies can be preferred to avoid these electromagnetic effects, such as slotless motors, torque motors having a large number of pole pairs, or brushed DC motors with commutator.

Capstan systems are designed to transmit the torque from the motors to the active joints. The smallest couple of actuator and capstan radius $r_{c a p, i}$ adequate to verify the criteria in Equation (16) is selected for each Delta robot. The capstan transmission ratio is $R_{i}=\frac{r_{m o t, i}}{r_{c a p, i}}$.

$$
\tau_{\text {max }, \text { Deltai }} \leq C_{\text {mot }, i}^{\max } / R_{i}(a) \quad \tau_{\text {eff,Deltai }} \leq C_{\text {mot, }, i}^{\text {nom }} / R_{i}(b) \quad J_{\text {mot }, i} \geq J_{\text {eq,i }} / 20 \text { (c) }
$$

$J_{e q, i}$ is the apparent inertia of the load from the motor point of view. It is worth noting that this apparent inertia varies with the configuration of the robot. The kinetic energy matrix in joint space $\boldsymbol{A}_{\oplus}$ is deduced from the dynamic equation (Eq. (9)). By considering for each joint that the apparent inertia of the load is the diagonal term of this kinetic energy matrix, the equivalent inertia on the motor axis becomes:

$$
J_{e q, i}=R_{i}^{2} \boldsymbol{A}_{\oplus}(i, i)
$$

The maximum equivalent inertia of the load on the motor axis is calculated over the prescribed workspace for each Delta robot. The maximum design inertia ratio $J_{e q, i} / 20$ is plotted in Fig. 10 according to the capstan transmission ratio.

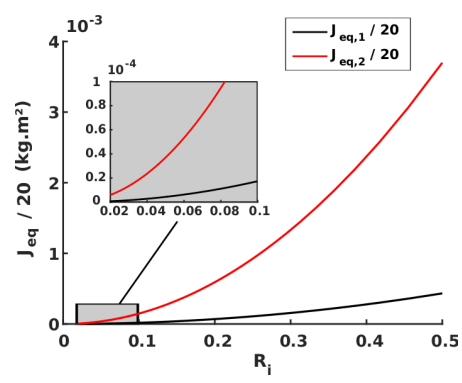

Figure 10: Maximum motor apparent inertia for each Delta robot 
The selection of the couple of actuators and capstan transmission systems for each Delta robot remains a designer choice as several solutions would check the criteria in Equation (16). The Table 4 explores solutions using brushed DC motors powered at $48 \mathrm{~V}$ nominal voltage. The transmission ratios $R_{i}$ are calculated for each motor by considering the smallest capstan radius allowing to check the three selection criteria. The limiting criterion $(a),(b)$, or $(c)$ is noted in the Table for each solution.

\begin{tabular}{ccccccccc} 
Table 4: Brushed DC motor and capstan transmission solutions \\
$\begin{array}{c}\text { Motor } \\
\text { designation }\end{array}$ & $\begin{array}{c}\emptyset_{\text {mot }} \\
{[\mathrm{mm}]}\end{array}$ & $\begin{array}{c}C_{m o x}^{\max } \\
{[\mathrm{Nm}]}\end{array}$ & $\begin{array}{c}C_{\text {mot }}^{\text {nom }} \\
{[\mathrm{Nm}]}\end{array}$ & $\begin{array}{c}J_{\text {mot }} \\
{\left[\mathrm{kg} . \mathrm{cm}^{2}\right]}\end{array}$ & $R_{1}$ & $\begin{array}{c}\text { Limiting } \\
\text { criteria }(1)\end{array}$ & $R_{2}$ & $\begin{array}{c}\text { Limiting } \\
\text { criteria (2) }\end{array}$ \\
\hline Maxon RE35 & 6 & 0.498 & 0.106 & 0.066 & $1 / 30$ & $(c)$ & $1 / 52$ & $(c)$ \\
Crouzet 89830 & 8 & 1.23 & 0.18 & 0.38 & $1 / 13$ & $(c)$ & $1 / 28$ & $(b)$ \\
Maxon RE40 & 6 & 0.814 & 0.19 & 0.12 & $1 / 22$ & $(c)$ & $1 / 39$ & $(c)$ \\
Crouzet 89890 & 8 & 2.68 & 0.29 & 0.65 & $1 / 10$ & $(c)$ & $1 / 18$ & $(b)$ \\
Maxon RE50 & 8 & 7.37 & 0.42 & 0.542 & $1 / 11$ & $(c)$ & $1 / 19$ & $(c)$ \\
Maxon RE65 & 12 & 16.1 & 0.8 & 1.34 & $1 / 7$ & $(c)$ & $1 / 12$ & $(c)$ \\
\hline
\end{tabular}

The results for the Delthaptic show that the requirements $(b)$ and $(c)$ about the effective torque and the inertia mismatch are key constraints in the selection procedure. They involve smaller transmission ratios (i.e. higher capstan radii) than the single condition of maximum joint performances.

Among the possible solutions, Maxon motors are selected for both Delta robots of the Delthaptic: the RE 40 for the first Delta with $R_{1}=1 / 22$ and the RE 50 with $R_{2}=1 / 19$ for the second one. This choice allows the development of identical driven pulleys for all capstan systems.

\section{Experimental validation}

A prototype of the Delthaptic has been produced in the lab to check the proper functioning of the mechanical structure and to validate the proposed design procedure.

\subsection{Prototype of the Delthaptic}

A first prototype of the Delthaptic is build and shown in Fig. 11. Its geometric parameters are chosen with respect to the results of the multi-objective optimization. Every R-joint is mounted on ball bearings which significantly reduces the mechanism friction. The parts are designed with openings and are made of aluminum alloy or carbon fiber to decrease their mass. Mechanical stoppers are installed on the actuated joints to prevent damages which could happen if the operator attempts to move outside the motion range.

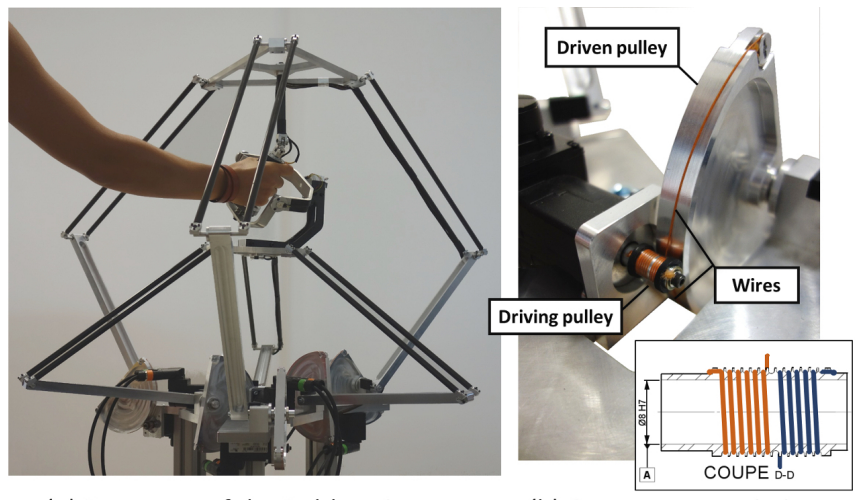

(a) Prototype of the Delthaptic

(b) Capstan transmission

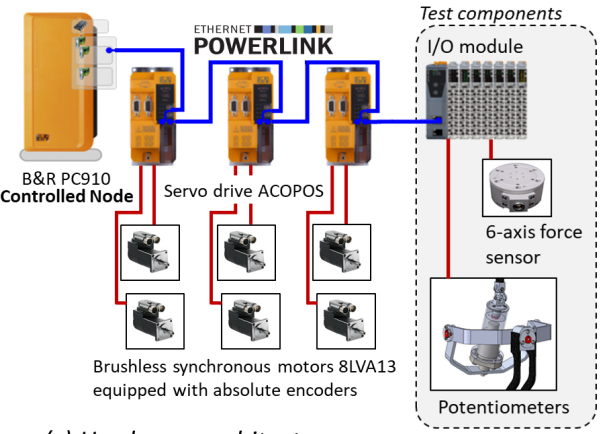

(c) Hardware architecture

Figure 11: First prototype and hardware architecture of the Delthaptic 
For this experimental device, available AC synchronous 8LVA1 motors from B\&R are used. Although this kind of brushless motors is not optimal, because they suffer from cogging torque, they allow to approve the design of the Delthatpic. Capstan systems providing a transmission ratio of $1 / 16$ are developed to ensure a sufficient force feedback according to the criteria of maximum and effective torques. Each transmission system is composed by an helical driving pulley (diameter: $10 \mathrm{~mm}$ ) fixed to the motor shaft, a flat half-disk driven pulley (diameter: $160 \mathrm{~mm}$ ) clamped to the Delta leg, and two $0.6 \mathrm{~mm}$-diameter vectran wires ensuring the system to be slack-free and stiff enough.

The hardware architecture of the haptic device is detailed in Fig. 11. To ensure a real-time control of all the components, a powerful Operating System and a high-speed robust communication with a wide bandwidth are required. The joints of the Delthaptic are driven by B\&R Automation industrial PC and ACOPOS servo drives running the inner current loop of the motors. An I/O module is temporary added to the control rack to evaluate the orientation of the handle thanks to potentiometers and the operational space force through a force sensor, during the experimental validation. The choice of Ethernet POWERLINK communication standard allows to synchronize the robot axes at a fast communication rate of $400 \mu s$. The programs cyclically compute the robot models and control schemes for the haptic application, including the evaluation of the set position of the slave robot and an open-loop implementation of the direct force feedback. The computation times are low and compatible with the communication rate: an average net time of $22 \mu \mathrm{s}$ for the axis manager, and $76 \mu \mathrm{s}$ for the application controller. The axis manager is based on the RTRobMultiAxisControl library, an open robotic real-time framework developed by the RoBioSS team with respect to PLCopen Motion and IEC 61131 standards. To specifically assess the prototype performances, a standard open-loop impedance controller generates the set value to the motor torque control loop.

\subsection{Prescribed workspace feedback}

A virtual perception of the operating space is created to prevent the user from getting out of the prescribed translational workspace and meeting a singular configuration. Virtual walls delimiting the boundaries of the operating area are rendered through the force feedback. Simple spring models with a stiffness $K=0.5 \mathrm{~N} / \mathrm{mm}$ are considered to provide these virtual walls.

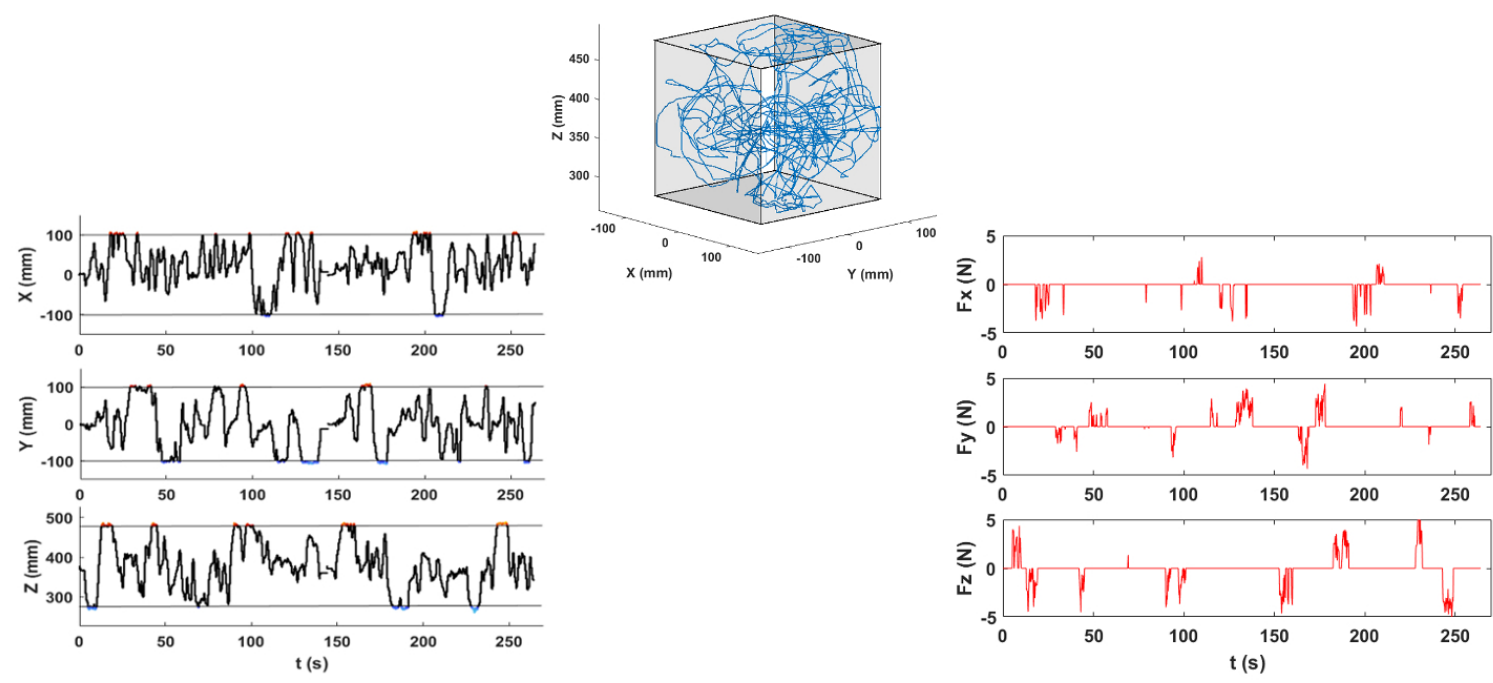

Figure 12: Free path of the end-effector with virtual workspace force feedback

The Fig. 12 displays an experimental path of a user moving freely the interface. The torque control compensates for the gravity effects on the Delthaptic and provides the feeling of the virtual operating space. The force feedback, represented in Fig. 12 along the path, constrains the operator motion inside the prescribed translational workspace. This perception of the prescribed workspace qualitatively highlights the ability of the Delthaptic to provide a force feedback. The resulting path also demonstrates the accessibility of the prescribed workspace as expected by the design optimization. 


\subsection{Positioning accuracy}

To estimate the positioning accuracy of the haptic device, the position in the operational space is evaluated thanks to a motion capture experiment with a Vicon system composed of 19 T40 cameras sampled at $100 \mathrm{~Hz}$. The Figure 13 details the experimental set-up and the placement of the markers on the Delthaptic.

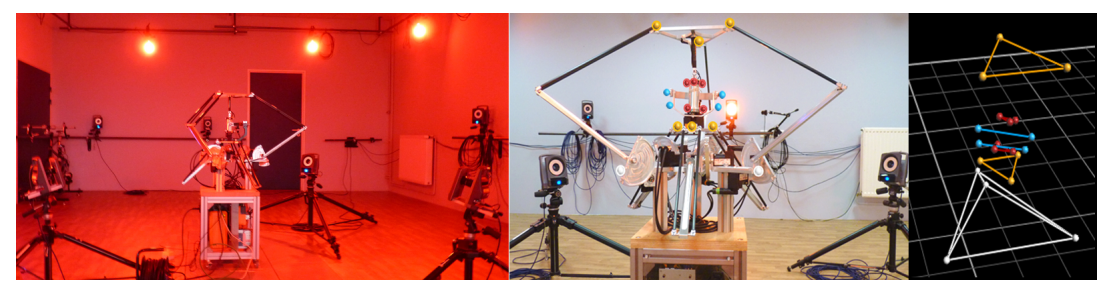

Figure 13: Motion capture experimental set-up

Two additional potentiometers are placed on the gimbal mechanism to get the handle orientation (see Fig.11). The Delthaptic operational space position is measured along a free path of the user thanks to the motion capture system and the potentiometers. These experimental values are compared in Fig. 14 to the position evaluated with the forward kinematic model from the joint encoders' values. The different positions are consistent together. These experimental results indicate a good positioning accuracy of the haptic device. Small errors are observed on the evaluation of the handle orientation, but can be explained by some imprecision in the measurements, since they are differences between potentiometers and motion capture results.
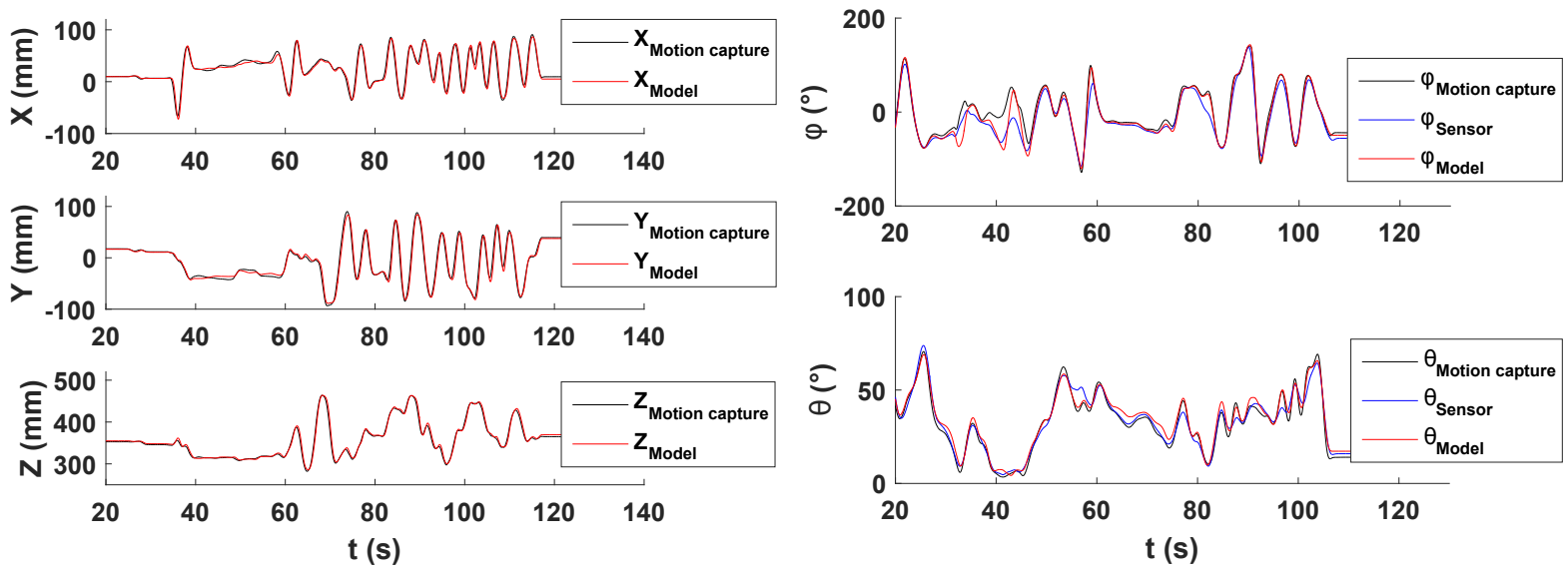

Figure 14: Position of the Delthaptic on a free path, model estimation and measured values

\subsection{Force feedback accuracy}

The accuracy of the force feedback is essential to ensure the transparency in contact of the haptic device. A six-axis force sensor is temporarily mounted on the handle to evaluate the force in operational space provided to the operator. The end-effector is virtually linked to the workspace center through a linear stiffness of $1800 \mathrm{~N} / \mathrm{m}$ in translation and $0.8 \mathrm{Nm} / \mathrm{rad}$ in rotation. The force feedback is measured while the operator is interacting with the virtual spring. The set force $F_{o p}$ and the measured force $F_{o p \text {,mes }}$ are compared in Fig. 15. $C_{Z o p}$ and $C_{Y o p}>0$ loads are not studied as the sensor wire restricts rotational motions in these directions.

The comparison of the set and measured forces in operational space shows a good accuracy of the haptic feedback during quasi-static interactions. A maximal mean deviation of $1 N$ is observed for a vertical solicitation of $\pm 10 N$, and can be explained by a non-perfect compensation of the gravity term. The average torque error reaches $0.05 \mathrm{Nm}$ for a torque feedback of $\pm 0.2 \mathrm{Nm}$. These mean errors are close to the requirement of force precision but would double 

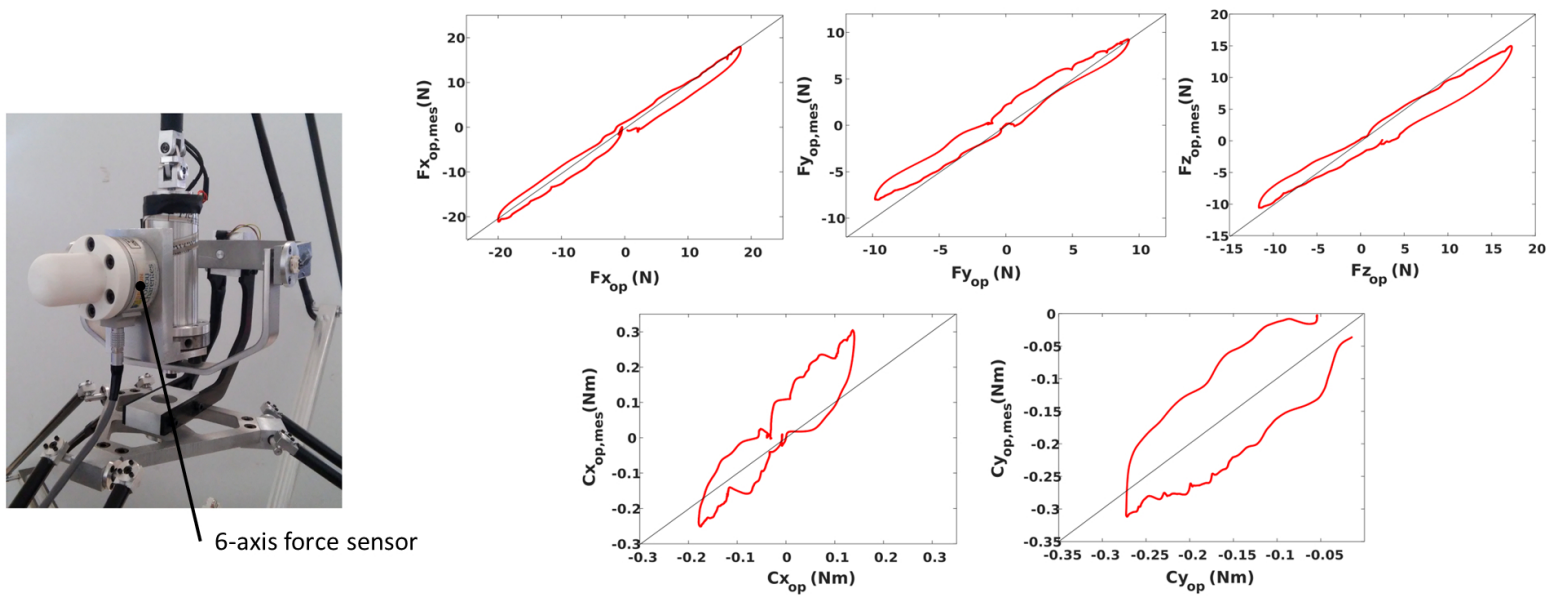

Figure 15: Evaluation of quasi-static force feedback

for the maximum force feedback. Moreover, a hysterisis phenomenon appears on these results, caused by dry friction and inducing additional force errors of $\pm 2 N$ and torque errors of $\pm 0.05 \mathrm{Nm}$. However, these errors in open-loop force control remain small and could easily be reduced by implementing a suitable closed-loop control strategy.

\subsection{Teleoperation application}

The Delthaptic has been integrated in a collaborative multi-robot cell to teleoperate a Racer 3 robot of COMAU for a simple grasping task. Real-time Ethernet POWERLINK communication is used to synchronize the robots. The haptic device switches between position and torque control modes with respect with the task state and the operator action. The Delthaptic compensates for the gravity effects and provides force feedback to the operator when teleoperating the COMAU robot. The communication structures in Fig. 16 are defined to exchange significant data for the process such as state information, numerical sensor data, or robot configuration data. The set and measured articular positions of the COMAU robot are plotted and show the accuracy of the Delthaptic motion tracking in the teleoperation phase. These curves are displayed for the joint $q_{3}$ in Fig. 16.

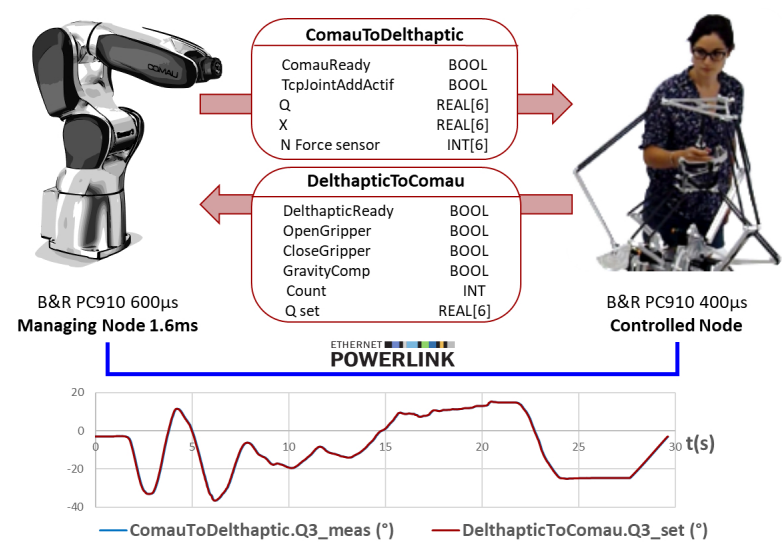

Figure 16: Teleoperation of a COMAU Racer 3 with the Delthaptic

A video of this application is available in the attached file "VideoDelthapticDextRobC": Integration of the Delthaptic in DextRobC, a multi-cobot cell (online version only). 


\section{Conclusion}

The design strategy of a new 6-DOF paired-parallel haptic device is presented in this paper. The Delthaptic provides a large and singularity-free workspace by linking two Delta robots to the handle. Its mechanical architecture possesses attributes of parallel structure valuable for the transparency of the interface such as low inertia, high stiffness, and high dynamics. The paired structure also presents simple kinematic and dynamic analytical models. To the authors's knowledge, the Delthaptic is the first fully parallel haptic device providing such a workspace, especially in rotation. This workspace is larger than those of available hybrid devices such as Sigma.7 of Force Dimension. In addition, the Delthaptic ensures an accurate haptic feedback within $\pm 20 \mathrm{~N}$ and $\pm 0.5 \mathrm{Nm}$. This force capability is crucial to properly replicate the task interactions with respect to human sensorimotor performances.

The geometric parameters of the Delthaptic are optimized by minimizing two kinematic objective functions. The actuators and transmission systems are chosen by following a selection procedure based on dynamic criteria and technological aspects. Testing of a prototype of the Delthaptic validates the expected functioning of the device and the design purposes. The conducted tests have demonstrated the ability of the device to teleoperate industrial and collaborative robots in remote tasks with force feedback.

As dynamic performance is crucial for haptic device, the actuator features, the parameters of the structure and the transmission systems are adjusted through a second optimization process based on dynamic objectives. This work is the subject of a second study.

\section{Acknowledgment}

This work is supported by the French national network of robotic platforms ROBOTEX (ANR10-EQPX-44-01). Declarations of interest: none.

\section{References}

[1] T. H. Massie, J. K. Salisbury, The phantom haptic interface: A device for probing virtual objects, in: Proceedings of the ASME winter annual meeting, symposium on haptic interfaces for virtual environment and teleoperator systems, Vol. 55, 1994, pp. 295-300. doi:10.1.1.361.4710.

[2] V. Hayward, P. Gregorio, O. Astley, S. Greenish, M. Doyon, L. Lessard, J. McDougall, I. Sinclair, S. Boelen, X. Chen, et al., Freedom7: A high fidelity seven axis haptic device with application to surgical training, in: Experimental Robotics V, 1998 , pp. 443-456. doi:10.1007/BFb0112983.

[3] R. Q. Van der Linde, P. Lammertse, E. Frederiksen, B. Ruiter, The hapticmaster, a new high-performance haptic interface, in: Proc. Eurohaptics, 2002, pp. 1-5.

[4] J. Lee, K. Eom, Design of a new 6-dof parallel haptic device, in: IEEE International Conference on Robotics and Automation, ICRA 2001, Vol. 1, 2001, pp. 886-891. doi:10.1109/ROBOT.2001.932662.

[5] P. Lambert, J. Herder, A novel parallel haptic device with 7 degrees of freedom, in: IEEE World Haptics Conference, WHC 2015, 2015, pp. 183-188. doi:10.1109/WHC.2015.7177711.

[6] Y. Tsumaki, H. Naruse, D. N. Nenchev, M. Uchiyama, Design of a compact 6-dof haptic interface, in: IEEE International Conference on Robotics and Automation, ICRA 1998, Vol. 3, 1998, pp. 2580-2585. doi:10.1109/ROBOT.1998.680730.

[7] J. Arata, H. Kondo, N. Ikedo, H. Fujimoto, Haptic device using a newly developed redundant parallel mechanism, IEEE Transactions on Robotics 27 (2) (2011) 201-214. doi:10.1109/TRO.2010.2098272.

[8] R. Clavel, Delta, a fast robot with parallel geometry, in: 18th International Symposium on Industrial Robots, 1988, pp. 91-100.

[9] D. Ryu, J.-B. Song, C. Cho, S. Kang, M. Kim, Development of a six dof haptic master for teleoperation of a mobile manipulator, Mechatronics 20 (2) (2010) 181-191. doi:10.1016/j.mechatronics.2009.11.003.

[10] S. S. Lee, J. M. Lee, Design of a general purpose 6-dof haptic interface, Mechatronics 13 (7) (2003) 697-722. doi:10.1016/S09574158(02)00038-7.

[11] J. Lallemand, A. Goudali, S. Zeghloul, The 6-dof 2-delta parallel robot, Robotica 15 (04) (1997) 407-416. doi:10.1017/S0263574797000507.

[12] F. Gosselin, C. Bidard, J. Brisset, Design of a high fidelity haptic device for telesurgery, in: IEEE International Conference on Robotics and Automation, ICRA 2005, 2005, pp. 205-210. doi:10.1109/ROBOT.2005.1570120.

[13] L.-F. Lee, M. S. Narayanan, F. Mendel, V. N. Krovi, Kinematics analysis of in-parallel 5 dof haptic device, in: IEEE/ASME International Conference on Advanced Intelligent Mechatronics, 2010, pp. 237-241. doi:10.1109/AIM.2010.5695908.

[14] G. Lee, S.-M. Hur, Y. Oh, High force-display capability and wide workspace with a novel haptic interface, IEEE/ASME Transactions on Mechatronicsdoi:10.1109/TMECH.2016.2624263.

[15] H. Bassan, A. Talasaz, R. Patel, Design and characterization of a 7-dof haptic interface for a minimally invasive surgery test-bed, in: IEEE/RSJ International Conference on Intelligent Robots and Systems, IROS 2009, 2009, pp. 4098-4103. doi:10.1109/IROS.2009.5354211.

[16] H. Z. Tan, M. A. Srinivasan, B. Eberman, B. Cheng, Human factors for the design of force-reflecting haptic interfaces, Dynamic Systems and Control 55 (1) (1994) 353-359.

[17] L. Jandura, M. A. Srinivasan, Experiments on human performance in torque discrimination and control, Dynamic systems and control, ASME, DSC-55 1 (1994) 369-375. 
[18] F. Pierrot, C. Reynaud, A. Fournier, Delta: a simple and efficient parallel robot, Robotica 8 (02) (1990) 105-109. doi:10.1017/S0263574700007669.

[19] M. Vulliez, S. Zeghloul, O. Khatib, Kinematic analysis of the delthaptic, a new 6-dof haptic device, in: Advances in Robot Kinematics 2016, 2018, pp. 181-189. doi:10.1007/978-3-319-56802-7_19.

[20] D. Zlatanov, I. A. Bonev, C. M. Gosselin, Constraint singularities of parallel mechanisms, in: IEEE International Conference on Robotics and Automation, ICRA 2002, Vol. 1, 2002, pp. 496-502. doi:10.1109/ROBOT.2002.1013408.

[21] W. Khalil, O. Ibrahim, General solution for the dynamic modeling of parallel robots, Journal of intelligent and robotic systems 49 (1) (2007) 19-37. doi:10.1007/s10846-007-9137-x.

[22] K.-S. Chang, R. Holmberg, O. Khatib, The augmented object model: Cooperative manipulation and parallel mechanism dynamics, in: IEEE International Conference on Robotics and Automation, ICRA 2000, Vol. 1, 2000, pp. 470-475. doi:10.1109/ROBOT.2000.844099.

[23] F. Hao, J.-P. Merlet, Multi-criteria optimal design of parallel manipulators based on interval analysis, Mechanism and machine theory 40 (2) (2005) 157-171. doi:10.1016/j.mechmachtheory.2004.07.002.

[24] M. Laribi, L. Romdhane, S. Zeghloul, Analysis and dimensional synthesis of the delta robot for a prescribed workspace, Mechanism and machine theory 42 (7) (2007) 859-870. doi:10.1016/j.mechmachtheory.2006.06.012.

[25] Z. Wang, S. Ji, Y. Wan, C. Ou, J. Sun, G. Wang, Optimal design of parallel robots for the prescribed regular dexterous workspace, in: IEEE International Conference on Automation and Logistics, 2007, pp. 563-568. doi:10.1109/ICAL.2007.4338628.

[26] M. Arsenault, R. Boudreau, The synthesis of three-degree-of-freedom planar parallel mechanisms with revolute joints (3-rrr) for an optimal singularity-free workspace, Journal of Robotic Systems 21 (5) (2004) 259-274. doi:10.1002/rob.20013.

[27] Z. Gao, D. Zhang, Y. Ge, Design optimization of a spatial six degree-of-freedom parallel manipulator based on artificial intelligence approaches, Robotics and Computer-Integrated Manufacturing 26 (2) (2010) 180-189. doi:10.1016/j.rcim.2009.07.002.

[28] S. Bai, Optimum design of spherical parallel manipulators for a prescribed workspace, Mechanism and Machine Theory 45 (2) (2010) 200-211. doi:10.1016/j.mechmachtheory.2009.06.007.

[29] R. Unal, G. Kiziltas, V. Patoglu, Multi-criteria design optimization of parallel robots, in: IEEE Conference on Robotics, Automation and Mechatronics, 2008, 2008, pp. 112-118. doi:10.1109/RAMECH.2008.4681427.

[30] J.-P. Merlet, Jacobian, manipulability, condition number, and accuracy of parallel robots, Journal of Mechanical Design 128 (1) (2006) 199-206. doi:10.1115/1.2121740.

[31] E. Courteille, D. Deblaise, P. Maurine, Design optimization of a delta-like parallel robot through global stiffness performance evaluation, in: IEEE/RSJ International Conference on Intelligent Robots and Systems, IROS 2009, 2009, pp. 5159-5166. doi:10.1109/IROS.2009.5353906.

[32] P. Chedmail, M. Gautier, Optimum choice of robot actuators, ASME Journal of Engineering for Industry 112 (4) (1990) $361-367$. doi:10.1115/1.2899600.

[33] A. Bowling, O. Khatib, Actuator selection for desired dynamic performance, in: IEEE/RSJ International Conference on Intelligent Robots and Systems, IROS 2002, Vol. 2, 2002, pp. 1966-1973. doi:10.1109/IRDS.2002.1044044.

[34] K. Vlachos, E. Papadopoulos, Transparency maximization methodology for haptic devices, IEEE/ASME Transactions on Mechatronics 11 (3) (2006) 249-255. doi:10.1109/TMECH.2006.875561.

[35] L. Zhou, S. Bai, M. R. Hansen, Integrated dimensional and drive-train design optimization of a light-weight anthropomorphic arm, Robotics and Autonomous Systems 60 (1) (2012) 113-122. doi:10.1016/j.robot.2011.09.004.

[36] M. Pettersson, J. Ölvander, Drive train optimization for industrial robots, IEEE Transactions on Robotics 25 (6) (2009) $1419-1424$. doi:10.1109/TRO.2009.2028764.

[37] R. W. Armstrong, Load to motor inertia mismatch: Unveiling the truth, in: Drives and Controls Conference, 1998.

[38] J. Moscrop, C. Cook, P. Moll, Control of servo systems in the presence of motor-load inertia mismatch, in: The 27th IEEE Conference on Industrial Electronics Society IECON 2001, 2001, pp. 351-356. doi:10.1109/IECON.2001.976507.

[39] A. D. Poole, J. D. Booker, Design methodology and case studies in actuator selection, Mechanism and machine theory 46 (5) (2011) 647-661. doi:10.1016/j.mechmachtheory.2010.12.009.

[40] Y. Zhang, J. Zhu, Direct torque control of permanent magnet synchronous motor with reduced torque ripple and commutation frequency, IEEE Transactions on Power Electronics 26 (1) (2011) 235-248. doi:10.1109/TPEL.2010.2059047.

[41] L. Tang, L. Zhong, M. F. Rahman, Y. Hu, A novel direct torque controlled interior permanent magnet synchronous machine drive with low ripple in flux and torque and fixed switching frequency, IEEE Transactions on power electronics 19 (2) (2004) $346-354$. doi:10.1109/TPEL.2003.823170. 